\title{
Luminescent Organoboron Quinolate Polymers
}

\author{
Yang Qin, Cynthia Pagba, Piotr Piotrowiak, and Frieder Jäkle* \\ Department of Chemistry, Rutgers University-Newark, 73 Warren Street, Newark, NJ 07102
}

\section{Supplementary Material}

Materials and General Methods. The compounds 8-hydroxyquinoline (99\%), thiophene (99+\%), N,N,N',N'-tetramethylethylenediamine (TMEDA; 99\%), $n$-BuLi (1.6M in hexanes) and 1-bromohexane $(99+\%)$ were purchased from Acros, trimethyltin chloride $(98 \%)$ was obtained from Strem Chemicals, and 3-bromothiophene (97\%) and $\mathrm{BBr}_{3}(99+\%)$ were purchased from Aldrich. 8-Hydroxyquinoline was sublimed and $\mathrm{BBr}_{3}$ was distilled under high vacuum prior to use. All other chemicals were used as received without further purification. The compounds poly(4-trimethylsilylstyrene) ${ }^{1}$, 4-dibromoborylcumene ${ }^{1}$, and 2-bromo-3-hexylthiophene ${ }^{2}$ were prepared as previously described. 2-Hexylthiophene was obtained by adaptation of a literature procedure for the synthesis of 2-(bromohexyl)thiophene ${ }^{3}$. Reactions and manipulations involving reactive boron species were carried out under an atmosphere of prepurified nitrogen using either Schlenk techniques or an inert-atmosphere glove box (Innovative Technologies). Ether solvents were distilled from Na/benzophenone prior to use. Hydrocarbon and chlorinated solvents were purified using a solvent purification system (Innovative Technologies; alumina / copper columns for hydrocarbon solvents), and the chlorinated solvents were subsequently distilled from $\mathrm{CaH}_{2}$ and degassed via several freeze pump thaw cycles.

Caution! $\mathrm{BBr}_{3}$ is toxic and highly corrosive and should be handled appropriately with great care. Fluorinated grease was used for ground glass joints in all reactions involving boron tribromide.

The $399.95 \mathrm{MHz}{ }^{1} \mathrm{H}$ and $100.5 \mathrm{MHz}{ }^{13} \mathrm{C}$ NMR spectra were recorded on a Varian VXR-S and the $499.91 \mathrm{MHz}{ }^{1} \mathrm{H}$ and $125.68 \mathrm{MHz}{ }^{13} \mathrm{C}$ NMR spectra were obtained on a Varian INOVA NMR spectrometer equipped with a $5 \mathrm{~mm}$ dual broadband gradient probe (Nalorac, Varian Inc., Martinez, CA). ${ }^{11} \mathrm{~B}$ NMR and ${ }^{119}$ Sn NMR data were collected on the VXR-S spectrometer at 128.3 MHz and 149.07 MHz, respectively. All NMR data were obtained at ambient temperature. ${ }^{1} \mathrm{H}$ and ${ }^{13} \mathrm{C}$ NMR spectra were referenced internally to the solvent peaks and ${ }^{11} \mathrm{~B}$ and ${ }^{119} \mathrm{Sn}$ NMR spectra were referenced externally to $\mathrm{BF}_{3} \cdot \mathrm{Et}_{2} \mathrm{O}(\square=0)$ and $\mathrm{SnMe}_{4}(\square=0)$ in $\mathrm{C}_{6} \mathrm{D}_{6}$, respectively. The abbreviations $\mathrm{Th}$ and $\mathrm{Q}$ are used for thienyl and quinolato, respectively. Two-dimensional proton correlation spectroscopy $\left(\mathrm{COSY}^{4,5}\right)$, and one bond proton-carbon correlation spectroscopy $\left(\mathrm{HMQC}^{6,7}\right)$ measurements were recorded on the INOVA spectrometer in the absolute value mode (COSY) or the phase sensitive mode (HMQC) by employing the TPPI improvement ${ }^{8,9}$ of the States-Haberkorn-Ruben Hypercomplex method ${ }^{10}$. Selection of desirable coherences and artifact 
suppression were accomplished by z-gradients (COSY, echo N-type coherence selection ${ }^{11}$ ), or phase cycles of 16 steps (HMQC). The HMQC pulse sequence included the BIRD filter to suppress signals from $\mathrm{C} 12$-attached protons ${ }^{12}$ and C13-decoupling during proton acquisition, using a $3.6 \mathrm{kHz}$ field strength and the GARP decoupling scheme ${ }^{13}$. Typically, $256 \mathrm{t} 1$ increments of $2 \mathrm{~K}$ complex data points over $5.5 \mathrm{kHz}$ (proton) and $22.5 \mathrm{kHz}$ (carbon) spectral widths were collected with 1 (COSY) or 16 (HMQC) scans per t1 increment, preceded by 16 or 32 dummy scans, and a relaxation delay of $2 \mathrm{~s}$. Baseline distortion was addressed by properly adjusting the sampling delay and the signal phase ${ }^{14}$. Data sets were processed on a Sun Blade 100 workstation (Sun Microsystems Inc., Palo Alto, CA) using the VNMR software package (Varian Inc., Palo Alto, CA). In order to decrease $t 1$ ridges arising from incorrect treatment of the first data point in the discreet Fourier transform (FT) algorithm, the spectrum corresponding to the first t1 value was divided by 2 prior to FT along t $1 .{ }^{15}$ Shifted (COSY) or unshifted (HMQC) Gaussian window functions were used in both dimensions. Data sets were zero-filled in the $\mathrm{t} 1$ dimension yielding $1 \mathrm{~K} \times 1 \mathrm{~K}$ final matrices that have not been symmetrized.

Elemental analyses were performed by Quantitative Technologies Inc., Whitehouse, NJ. Mass spectral data (FAB mode with either 3-nitrobenzylalcohol (NBA) or 2-nitrophenyloctyl ether (NPOE) as the matrix) were obtained at the Michigan State University Mass Spectrometry Facility which is supported, in part, by a grant (DRR-00480) from the Biotechnology Research Technology Program, National Center for Research Resources, National Institutes of Health.

GPC analyses were performed in THF $(1 \mathrm{ml} / \mathrm{min})$ using a Waters Breeze system equipped with a 717plus autosampler, a 1525 binary HPLC pump, a 2487 dual $\square$ absorbance detector, and a 2414 refractive index detector. A series of styragel columns (Polymer Laboratories; $5 \square \mathrm{m}$ MixD, $5 \square \mathrm{m} \mathrm{Mix-C}$, and $10 \square \mathrm{m}$ Mix-B), which were kept in a column heater at $35^{\circ} \mathrm{C}$, were used for separation. The columns were calibrated with polystyrene standards (Polymer Laboratories). Multi Angle Laser Light Scattering (MALLS) experiments were performed at $690 \mathrm{~nm}(30 \mathrm{~mW}$ linear polarized GaAs laser) using a Wyatt Dawn EOS instrument either in a batch mode or inline with the GPC as specified; differential refractive indices $d n / d c$ were determined using a Wyatt Optilab at $690 \mathrm{~nm}$. DSC measurements were performed on a Perkin Elmer Differential Scanning Calorimeter Pyris 1 system with ca. $20 \mathrm{mg}$ of polymer and at a scan rate of $20{ }^{\circ} \mathrm{C} /$ min. The reported results correspond to the second heating cycle.

UV-visible measurements were performed in THF using a Varian Cary 500 scan UV-Vis-NIR spectrophotometer with a $1 \mathrm{~cm}$ quartz cuvette. The fluorescence data and quantum yields were measured on a Varian Cary Eclipse Fluorescence spectrophotometer with the same solutions as those used in the UV-visible measurements. Anthracene was used as the standard for determination of the quantum yields. The quantum yield of anthracene was adopted from the 'Handbook of Photochemistry, $2^{\text {nd }}$ Edition, Revised and Expanded. ISBN 0-8247-7911-8' and the concentration of anthracene in THF was $6.86 \cdot 10^{-6} \mathrm{M}$. For the luminescence lifetime measurements thoroughly degassed samples of the polymer and model compound were excited with $5 \square \mathrm{s} \sim 100 \mathrm{~mJ}$ pulses at $355 \mathrm{~nm}$ ( $3^{\text {rd }}$ harmonic of a Q-switched Nd:YAG laser, Quantel, Brilliant). The emission was dispersed through a monochromator (Oriel M257) and detected with a Hamamatsu R928 photomultiplier. The transients were recorded using a Tektronix SCD 1000 digital oscilloscope controlled by a Labview subroutine. Fluorescent decays were fitted as single exponentials using the Igor software package by Wavemetrics, Inc. 


\section{Synthesis of Precursors and Model Compounds}

Synthesis of 2-Hexylthiophene. 2-Hexylthiophene was obtained by adaptation of a literature procedure for the synthesis of 2-(bromohexyl)thiophene. ${ }^{3}$ Thiophene $(20.0 \mathrm{~g}, 0.238 \mathrm{~mol})$ and TMEDA (30.4 g, $0.262 \mathrm{~mol})$ were dissolved in $250 \mathrm{ml}$ THF, and $n$-BuLi (164 ml, $1.6 \mathrm{M}$ in hexanes, $0.262 \mathrm{~mol}$ ) was added dropwise at room temperature. The mixture was stirred at room temperature for $2 \mathrm{~h}$. 1-Bromohexane $(40 \mathrm{ml}, 47.2 \mathrm{~g}, 0.286 \mathrm{~mol})$ was added dropwise and the mixture was then heated to a gentle reflux and stirred for $8 \mathrm{~h}$. After quenching with ice water and aqueous work-up, the product was distilled at $55^{\circ} \mathrm{C}$ under high vacuum. 2-Hexylthiophene was obtained as a colorless liquid (23.0 g, $58 \%$ yield). ${ }^{1} \mathrm{H}$ NMR $\left(499.893 \mathrm{MHz}, \mathrm{CDCl}_{3}\right): \square=7.12(\mathrm{~d}$, $J=4.0 \mathrm{~Hz}, 1 \mathrm{H}$, Th-H3), 6.93 (dd, $J=2.5 \mathrm{~Hz} / 4.0 \mathrm{~Hz}, 1 \mathrm{H}$, Th-H4), 6.79 (d, $J=2.5 \mathrm{~Hz}, 1 \mathrm{H}$, ThH5), $2.83\left(\mathrm{t}, J=7.5 \mathrm{~Hz}, 2 \mathrm{H}\right.$, Th- $\left.\mathrm{CH}_{2}\right), 1.67(\mathrm{~m}, 2 \mathrm{H}$, hexyl-H), $1.35(\mathrm{~m}, 6 \mathrm{H}$, hexyl-H), $0.90(\mathrm{t}, J=$ $\left.7.0 \mathrm{~Hz}, 3 \mathrm{H}, \mathrm{CH}_{3}\right)$.

Synthesis of 5-Hexyl-2-trimethylstannylthiophene. 2-Hexylthiophene (23.0 g, $0.137 \mathrm{~mol})$ was dissolved in $250 \mathrm{ml}$ THF and $n$-BuLi ( $94 \mathrm{ml}, 1.6 \mathrm{M}$ in hexanes, $0.150 \mathrm{~mol}$ ) was added dropwise at $0{ }^{\circ} \mathrm{C}$. The mixture was stirred at $0{ }^{\circ} \mathrm{C}$ for $1 \mathrm{~h}$ and then cooled down to $-78{ }^{\circ} \mathrm{C}$. A solution of trimethyltin chloride $(32.8 \mathrm{~g}, 0.164 \mathrm{~mol})$ in $100 \mathrm{ml}$ THF was added dropwise. The mixture was allowed to warm slowly to room temperature and stirred for $2 \mathrm{~h}$. After quenching with ice water and aqueous work-up, the product was distilled at $108{ }^{\circ} \mathrm{C}$ under high vacuum. 5Hexyl-2-trimethylstannylthiophene was obtained as a colorless liquid $\left(40.8 \mathrm{~g}, 90 \%\right.$ yield). ${ }^{1} \mathrm{H}$ NMR (399.952 MHz, CDCl $)$ : $\square=7.03\left(\mathrm{~d}, J=3.2 \mathrm{~Hz}, J\left({ }^{19 / 117} \mathrm{Sn}, \mathrm{H}\right)=27 \mathrm{~Hz}, 1 \mathrm{H}, \mathrm{Th}-\mathrm{H} 3\right), 6.91$ $(\mathrm{d}, J=3.2 \mathrm{~Hz}, 1 \mathrm{H}, \mathrm{Th}-\mathrm{H} 4), 2.86\left(\mathrm{t}, J=7.6 \mathrm{~Hz}, 2 \mathrm{H}\right.$, Th- $\left.\mathrm{CH}_{2}\right), 1.69$ (m, 2H, hexyl-H), 1.34 (m, $6 \mathrm{H}$, hexyl-H), 0.90 (t, $\left.J=7.0 \mathrm{~Hz}, 3 \mathrm{H}, \mathrm{CH}_{3}\right), 0.35\left(\mathrm{~s} / \mathrm{d}, J\left({ }^{119 / 117} \mathrm{Sn}, \mathrm{H}\right)=58 \mathrm{~Hz} / 55 \mathrm{~Hz}, 9 \mathrm{H}, \mathrm{Sn}-\mathrm{Me}\right)$; ${ }^{13} \mathrm{C} \mathrm{NMR}\left(125.698 \mathrm{MHz}, \mathrm{CDCl}_{3}\right)$ : $\square=152.1$ (Th-C5), $135.2\left(\mathrm{~s} / \mathrm{d}, J\left({ }^{11 / 117} \mathrm{Sn}, \mathrm{C}\right)=30 \mathrm{~Hz}, \mathrm{Th}-\mathrm{C} 3\right)$, 134.8 (Th-C2), $125.6\left(\mathrm{~s} / \mathrm{d}, J\left({ }^{19 / 117} \mathrm{Sn}, \mathrm{C}\right)=42 \mathrm{~Hz}, \mathrm{Th}-\mathrm{C} 4\right), 32.1,31.8,30.2,29.2,22.8,14.3$ $\left(\right.$ hexyl-C), $-8.1\left(\mathrm{~s} / \mathrm{d}, J\left({ }^{119 / 117} \mathrm{Sn}, \mathrm{C}\right)=369 \mathrm{~Hz} / 353 \mathrm{~Hz}, \mathrm{Sn}\left[\mathrm{CH}_{3}\right]_{3}\right) ;{ }^{119} \mathrm{Sn} \mathrm{NMR}(149.071 \mathrm{MHz}$, $\left.\mathrm{CDCl}_{3}\right): \square=-27.4$.

Synthesis of 4-[8-Hydroxyquinolato-2-(5-hexylthienyl)]borylcumene (M-B5HTQ). 4-Dibromoborylcumene $(0.50 \mathrm{~g}, 1.73 \mathrm{mmol})$ was dissolved in $20 \mathrm{ml} \mathrm{CHCl}_{3}$ and a solution of 5-hexyl2-trimethylstannylthiophene $(1.14 \mathrm{~g}, 3.45 \mathrm{mmol})$ in $10 \mathrm{ml} \mathrm{CHCl}_{3}$ was added dropwise. The mixture was stirred at room temperature for $2 \mathrm{~h}$. Conversion to M-B5HT was confirmed by NMR spectroscopy and the product was used without further purification. For M-B5HT: ${ }^{1} \mathrm{H}$ NMR (499.893 MHz, $\left.\mathrm{CDCl}_{3}\right): \square=7.72(\mathrm{~d}, J=8.0 \mathrm{~Hz}, 2 \mathrm{H}, \mathrm{Ph}-\mathrm{H} 2,6), 7.67$ (d, $J=3.2 \mathrm{~Hz}, 2 \mathrm{H}$, Th-H3), 7.34 (d, $J=8.0 \mathrm{~Hz}, 2 \mathrm{H}, \mathrm{Ph}-\mathrm{H} 3,5), 7.06$ (d, $J=3.2 \mathrm{~Hz}, 2 \mathrm{H}$, Th-H4), 3.02 (hept, 1H, $J=$ $\left.7.0 \mathrm{~Hz}, \mathrm{CH}\left[\mathrm{CH}_{3}\right]_{2}\right), 1.36\left(\mathrm{~d}, J=7.0 \mathrm{~Hz}, 6 \mathrm{H}, \mathrm{CH}\left[\mathrm{CH}_{3}\right]_{2}\right), 2.97$ (t, $\left.J=7.6 \mathrm{~Hz}, 4 \mathrm{H}, \mathrm{Th}-\mathrm{CH}_{2}\right), 1.79$ $\left(\mathrm{m}, 4 \mathrm{H}\right.$, hexyl-H), $1.44\left(\mathrm{~m}, 12 \mathrm{H}\right.$, hexyl-H), 0.93 (t, $J=7.2 \mathrm{~Hz}, 6 \mathrm{H}$, hexyl-H); ${ }^{13} \mathrm{C}$ NMR $(125.698$ $\mathrm{MHz}, \mathrm{CDCl}_{3}$ ): $\square=158.9$ (Th-C5), 151.0 (Ph-C4), 143.3 (br., Th-C2), 143.0 (Th-C3), 141.2 (br., Ph-C1), 136.8 (Ph-C2,6), 126.9 (Th-C4), $125.5(\mathrm{Ph}-\mathrm{C} 3,5), 34.4\left(\mathrm{CH}\left[\mathrm{CH}_{3}\right]_{2}\right), 24.1\left(\mathrm{CH}\left[\mathrm{CH}_{3}\right]_{2}\right)$, $31.8,31.7,30.8,29.2,22.8,14.3$ (hexyl-C). A solution of 8-hydroxyquinoline $(0.25 \mathrm{~g}, 1.73$ $\mathrm{mmol}$ ) in $10 \mathrm{ml} \mathrm{CHCl}_{3}$ was added dropwise to the reaction mixture. The mixture turned yellow immediately with green luminescence and was kept stirring for $2 \mathrm{~h}$. After removal of all volatile components, the product was recrystallized twice from a mixture of $n$-hexane and chloroform (ca. 2:1 by volume). M-B5HTQ was obtained as a yellow solid (0.61 g, $80 \%$ yield). For MB5HTQ: ${ }^{1} \mathrm{H}$ NMR $\left(499.893 \mathrm{MHz}, \mathrm{CDCl}_{3}\right)$ : $\square=8.62(\mathrm{~d}, J=5.0 \mathrm{~Hz}, 1 \mathrm{H}, \mathrm{Q}-\mathrm{H} 2), 8.39(\mathrm{~d}, J=8.0$ $\mathrm{Hz}, 1 \mathrm{H}, \mathrm{Q}-\mathrm{H} 4), 7.68$ (dd, $J=8.5 \mathrm{~Hz} / 8.0 \mathrm{~Hz}, 1 \mathrm{H}, \mathrm{Q}-\mathrm{H} 6), 7.61$ (dd, $J=8.5 \mathrm{~Hz} / 5.0 \mathrm{~Hz}, 1 \mathrm{H}, \mathrm{Q}-\mathrm{H} 3$ ), $7.51(\mathrm{~d}, J=8.0 \mathrm{~Hz}, 2 \mathrm{H}, \mathrm{Ph}-\mathrm{H} 2,6), 7.24$ (d, $J=8.0 \mathrm{~Hz}, 1 \mathrm{H}, \mathrm{Q}-\mathrm{H} 5), 7.17$ (d, $J=8.0 \mathrm{~Hz}, 1 \mathrm{H}$, Q- 
H7), $7.16(\mathrm{~d}, J=8.0 \mathrm{~Hz}, 2 \mathrm{H}, \mathrm{Ph}-\mathrm{H} 3,5), 6.91(\mathrm{~d}, J=3.2 \mathrm{~Hz}, 1 \mathrm{H}, \mathrm{Th}-\mathrm{H} 3), 6.74(\mathrm{~d}, J=3.2 \mathrm{~Hz}, 1 \mathrm{H}$, Th-H4), 2.87 (hept, $\left.J=7.0 \mathrm{~Hz}, 1 \mathrm{H}, \mathrm{CH}\left[\mathrm{CH}_{3}\right]_{2}\right), 2.77$ (t, $J=8.0 \mathrm{~Hz}, 2 \mathrm{H}$, Th- $\left.\mathrm{CH}_{2}\right), 1.63(\mathrm{~m}, 2 \mathrm{H}$, hexyl-H), $1.34\left(\mathrm{~m}, 4 \mathrm{H}\right.$, hexyl-H), $1.28\left(\mathrm{~m}, 2 \mathrm{H}\right.$, hexyl-H), $1.23\left(\mathrm{~d}, J=7.0 \mathrm{~Hz}, 6 \mathrm{H}, \mathrm{CH}\left[\mathrm{CH}_{3}\right]_{2}\right)$, 0.87 (t, $J=7.0 \mathrm{~Hz}, 3 \mathrm{H}$, hexyl-H); ${ }^{13} \mathrm{C} \mathrm{NMR}\left(125.698 \mathrm{MHz}^{\mathrm{CDCl}} \mathrm{CDC}_{3}\right): \square=158.5$ (Q-C8), 147.7, 147.9 (Th-C5 and Ph-C4), 147.2 (br., Th-C2), 143.7 (br., Ph-C1), 139.5 (Q-C2), 138.8 (Q-C4), 137.1 (Q-C9), 132.8 (Q-C6), 131.8 (Ph-C2,6), 130.1 (Th-C3), 128.3 (Q-C10), 125.8 (Ph-C3,5), 124.8 (Th-C4), 122.9 (Q-C3), 112.5 (Q-C5), 109.7 (Q-C7), $34.0\left(\boldsymbol{C H}\left[\mathrm{CH}_{3}\right]_{2}\right), 24.2\left(\mathrm{CH}\left[\mathrm{CH}_{3}\right]_{2}\right)$, 31.9, 31.7, 30.3, 29.0, 22.7, 14.2 (hexyl-C); ${ }^{11} \mathrm{~B} \mathrm{NMR}\left(128.286 \mathrm{MHz}, \mathrm{CDCl}_{3}\right)$ : $\square=10.4\left(\mathrm{w}_{1 / 2}=\right.$

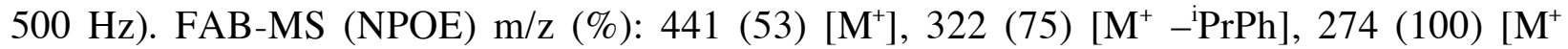
-HexTh]. Elemental analysis: calcd. C 76.18, H 7.31, N 3.17; found C 76.33, H 7.49, 3.02. The absence of any cumene in the crude product was confirmed by GC-MS analysis, which showed only one signal for 2-hexylthiophene: $\mathrm{m} / z=168(100 \%)$.

Synthesis of 3-Hexyl-2-trimethylstannylthiophene. To a Grignard solution prepared from 2bromo-3-hexylthiophene (21.6 g, $87.4 \mathrm{mmol})$ and $\mathrm{Mg}$ turnings $(4.25 \mathrm{~g}, 175 \mathrm{mmol})$ in $300 \mathrm{ml}$ THF was added dropwise a solution of trimethyltin chloride $(21.0 \mathrm{~g}, 105 \mathrm{mmol})$ in THF. The mixture was then heated to reflux overnight and subsequently quenched with ice water. Following standard workup, distillation $\left(10^{-3}\right.$ torr, $\left.85-86^{\circ} \mathrm{C}\right)$ gave $19.8 \mathrm{~g}(68 \%)$ of 3-Hexyl-2trimethylstannylthiophene as a colorless liquid. ${ }^{1} \mathrm{H}$ NMR $\left(499.893 \mathrm{MHz}, \mathrm{CDCl}_{3}\right): \square=7.59(\mathrm{~d}, J=$ $5.0 \mathrm{~Hz}, J\left({ }^{119 / 117} \mathrm{Sn}, \mathrm{H}\right)=13 \mathrm{~Hz}, 1 \mathrm{H}$, Th-H5), $7.17(\mathrm{~d}, J=5.0 \mathrm{~Hz}, 1 \mathrm{H}, \mathrm{Th}-\mathrm{H} 4), 2.71(\mathrm{t}, J=8.0 \mathrm{~Hz}$, 2H, Th- $\left.\mathrm{CH}_{2}\right), 1.67\left(\mathrm{~m}, 2 \mathrm{H}\right.$, hexyl-H), $1.39\left(\mathrm{~m}, 6 \mathrm{H}\right.$, hexyl-H), $0.98\left(\mathrm{t}, J=7.0 \mathrm{~Hz}, 3 \mathrm{H}, \mathrm{CH}_{3}\right), 0.46$ $\left(\mathrm{s} / \mathrm{d}, J\left({ }^{119 / 117} \mathrm{Sn}, \mathrm{H}\right)=57 \mathrm{~Hz} / 55 \mathrm{~Hz}, 9 \mathrm{H}, \mathrm{Sn}\left[\mathrm{CH}_{3}\right]_{3}\right) ;{ }^{13} \mathrm{C} \mathrm{NMR}\left(125.698 \mathrm{MHz}, \mathrm{CDCl}_{3}\right): \square=151.0$ (Th-C3), 131.5 (Th-C2), 130.7 (s/d, $J\left({ }^{119 / 117} \mathrm{Sn}, \mathrm{C}\right)=23 \mathrm{~Hz}$, Th-C4), $129.5\left(\mathrm{~s} / \mathrm{d}, J\left({ }^{119 / 117} \mathrm{Sn}, \mathrm{C}\right)=42\right.$ Hz, Th-C5), 32.8, 32.4, 32.0, 29.6, 22.8, 14.3 (hexyl), -7.8 (s/d, $J\left({ }^{119 / 117} \mathrm{Sn}, \mathrm{C}\right)=368 \mathrm{~Hz} / 351 \mathrm{~Hz}$, $\left.\mathrm{Sn}\left[\mathrm{CH}_{3}\right]_{3}\right)$.

Synthesis of 4-(8-Hydroxyquinolato-2-(3-hexylthienyl))borylcumene (M-B3HT). 4-Dibromoborylcumene $(0.50 \mathrm{~g}, 1.73 \mathrm{mmol})$ was dissolved in $20 \mathrm{ml} \mathrm{CH}_{2} \mathrm{Cl}_{2}$ and a solution of 3hexyl-2-trimethylstannylthiophene $(1.26 \mathrm{~g}, 3.80 \mathrm{mmol})$ in $10 \mathrm{ml} \mathrm{CH}_{2} \mathrm{Cl}_{2}$ was added dropwise. The mixture was stirred at room temperature for $48 \mathrm{~h}$. Conversion to M-B3HT was confirmed by NMR spectroscopy and the product was used without further purification. For M-B3HT: ${ }^{1} \mathrm{H}$ NMR (499.893 MHz, $\mathrm{CDCl}_{3}$ ): $\square=7.73$ (d, $J=5.0 \mathrm{~Hz}, 2 \mathrm{H}$, Th-H5), 7.59 (d, $J=7.5 \mathrm{~Hz}, 2 \mathrm{H}, \mathrm{Ph}-$ $\mathrm{H} 2,6), 7.26$ (d, $J=7.5 \mathrm{~Hz}, 2 \mathrm{H}, \mathrm{Ph}-\mathrm{H} 3,5), 7.16$ (d, $J=5.0 \mathrm{~Hz}, 2 \mathrm{H}, \mathrm{Th}-\mathrm{H} 4), 2.98$ (hept, $J=7.0 \mathrm{~Hz}$, $\left.1 \mathrm{H}, \mathrm{CH}\left[\mathrm{CH}_{3}\right]_{2}\right), 1.31\left(\mathrm{~d}, J=7.0 \mathrm{~Hz}, 6 \mathrm{H}, \mathrm{CH}\left[\mathrm{CH}_{3}\right]_{2}\right), 2.41\left(\mathrm{t}, J=7.5 \mathrm{~Hz}, 4 \mathrm{H}, \mathrm{Th}-\mathrm{CH}_{2}\right), 1.44(\mathrm{~m}$, 4H, hexyl-H), 1.17 (m, 4H, hexyl-H), $1.06(\mathrm{~m}, 8 \mathrm{H}$, hexyl-H), 0.78 (t, $J=7.0 \mathrm{~Hz}, 6 \mathrm{H}$, hexyl-H). A solution of 8-hydroxyquinoline $(0.28 \mathrm{~g}, 1.9 \mathrm{mmol})$ in $10 \mathrm{ml} \mathrm{CH}_{2} \mathrm{Cl}_{2}$ was added dropwise. The mixture was kept stirring for $24 \mathrm{~h}$. After removal of all volatile components, the product was recrystallized twice from a mixture of $n$-hexane and THF (ca. 3:1 by volume). The organoboron quinolate M-B3HTQ was obtained as a yellow solid $(0.35 \mathrm{~g}, 46 \%$ yield $) .{ }^{1} \mathrm{H}$ NMR (399.844 $\left.\mathrm{MHz}, \mathrm{CDCl}_{3}\right): \square=8.70(\mathrm{~d}, J=5.2 \mathrm{~Hz}, 1 \mathrm{H}, \mathrm{Q}-\mathrm{H} 2), 8.41(\mathrm{~d}, J=8.4 \mathrm{~Hz}, 1 \mathrm{H}, \mathrm{Q}-\mathrm{H} 4), 7.65$ (m, 2H, Q-H3 and Q-H6), $7.47(\mathrm{~d}, J=8.0 \mathrm{~Hz}, 2 \mathrm{H}, \mathrm{Ph}-\mathrm{H} 2,6), 7.25(\mathrm{~m}, 2 \mathrm{H}$, Th-H5 and Q-H5), 7.15 (m,

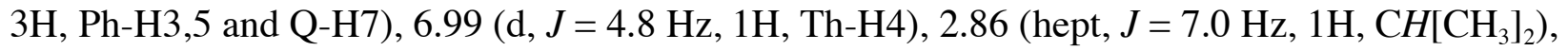
$2.40\left(\mathrm{~m}, 2 \mathrm{H}, \mathrm{Th}-\mathrm{CH}_{2}\right), 1.53(\mathrm{~m}, 2 \mathrm{H}$, hexyl-H), $1.33(\mathrm{~m}, 2 \mathrm{H}$, hexyl-H), $1.22(\mathrm{~d}, J=7.0 \mathrm{~Hz}, 6 \mathrm{H}$, $\left.\mathrm{CH}\left[\mathrm{CH}_{3}\right]_{2}\right), 1.00\left(\mathrm{~m}, 4 \mathrm{H}\right.$, hexyl-H), $0.82\left(\mathrm{t}, 3 \mathrm{H}, \mathrm{CH}_{3}\right) \cdot{ }^{13} \mathrm{C} \mathrm{NMR}\left(100.541 \mathrm{MHz}, \mathrm{CDCl}_{3}\right): \square=$ 158.7 (Q-C8), 147.7, (Ph-C4), 146.3 (Th-C3), 146.1 (br., Th-C2), 140.5 (br., Ph-C1), 140.1 (QC2), 139.1 (Q-C4), 137.7 (Q-C9), 133.1 (Q-C6), 131.7 (Ph-C2,6), 130.9 (Th-C5), 128.6 (QC10), 125.8 (Ph-C3,5), 125.5 (Th-C4), 122.9 (Q-C3), 112.5 (Q-C5), 110.1 (Q-C7), 34.1 $\left(\mathrm{CH}\left[\mathrm{CH}_{3}\right]_{2}\right), 24.2\left(\mathrm{CH}\left[\mathrm{CH}_{3}\right]_{2}\right), 31.9,31.8,30.7,29.6,22.8,14.3$ (hexyl-C). ${ }^{11} \mathrm{~B}$ NMR (128.286 
$\left.\mathrm{MHz}, \mathrm{CDCl}_{3}\right): \square=10.2\left(\mathrm{w}_{1 / 2}=400 \mathrm{~Hz}\right)$. FAB-MS (NPOE) $m / z(\%): 441(30)\left[\mathrm{M}^{+}\right], 322(82)\left[\mathrm{M}^{+}\right.$

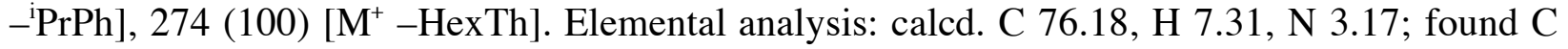
76.18, H 7.21, N 3.06.

\section{Synthesis of Polymers}

Synthesis of poly[4-(8-hydroxyquinolato-2-thienyl)borylstyrene] (PS-BTQ). A solution of PS-BBr prepared from $\mathrm{BBr}_{3}(3.11 \mathrm{~g} ; 12.4 \mathrm{mmol})$ and PS-Si $(2.0 \mathrm{~g}$; ca. $11.3 \mathrm{mmol}$ repeating units; $\left.M_{w}=26,000 ; P D I=1.13\right)$ in $20 \mathrm{ml} \mathrm{CH}_{2} \mathrm{Cl}_{2}$ was treated with a solution of 2(trimethylstannyl)thiophene $(6.10 \mathrm{~g} ; 28.3 \mathrm{mmol})$ in $\mathrm{CH}_{2} \mathrm{Cl}_{2}(10 \mathrm{ml})$ and the mixture was stirred for $12 \mathrm{~h}$. The reaction solution was concentrated under high vacuum to ca. $5 \mathrm{ml}$ and the polymer was recovered by repeated precipitation into hexanes. The resulting white solid was washed with hexanes and dried under high vacuum for $24 \mathrm{~h}$ at $50{ }^{\circ} \mathrm{C}$ to give $2.5 \mathrm{~g}(80 \%)$ PS-BT. For PS-BT: ${ }^{1} \mathrm{H}$ NMR (499.893 MHz, $\mathrm{CDCl}_{3}$ ) $\square=8.0-7.4$ (br., 6H, Ph-H2,6 and Th-H3,5), 7.08 (br., 2H, ThH4), 6.9-6.7 (br., 2H, Ph-H3,5), 2.6-1.4 (br. m, 3H, $\left.\mathrm{CH}_{2} \mathrm{CH}\right) ;{ }^{13} \mathrm{C} \mathrm{NMR} \mathrm{(125.681} \mathrm{MHz,} \mathrm{CDCl}_{3}$ ) $\square$ = 149.2, 148.4 (br., Ph-C4), 145.1 (Th-C2), 142.3 (Th-C5), 140.9 (br., Ph-C1), 137.4 (br., PhC2,6), 137.0 (Th-C3), 129.0 (Th-C4), 127.1 (br., Ph-C3,5), 47-42 (br., $\mathrm{CH}_{2} \mathrm{CH}$ ), 41 (br., $\left.\mathrm{CH}_{2} \mathrm{CH}\right) ;{ }^{11} \mathrm{~B}$ NMR $\left(160.370 \mathrm{MHz}, \mathrm{CDCl}_{3}\right) \square=50\left(\mathrm{w}_{1 / 2}=2,500 \mathrm{~Hz}\right)$. Elemental analysis: calcd. $\mathrm{C}$ 68.58, H 4.68; found C 68.17, H 4.50. PS-BT $(0.50 \mathrm{~g}, 1.78 \mathrm{mmol})$ was dissolved in $20 \mathrm{ml}$ $\mathrm{CH}_{2} \mathrm{Cl}_{2}$ and a solution of 8-hydroxyquinoline $(0.27 \mathrm{~g}, 1.87 \mathrm{mmol})$ in $10 \mathrm{ml} \mathrm{CH}_{2} \mathrm{Cl}_{2}$ was added dropwise. The mixture was stirred for $24 \mathrm{~h}$ and then concentrated to a small volume $(\sim 2 \mathrm{ml})$. The polymer PS-BTQ was recovered as a yellow solid by precipitation into hexanes (yield: $78 \%$ ). ${ }^{1} \mathrm{H}$ NMR (499.893 MHz, $\mathrm{CDCl}_{3}$ ): $\square=8.5-6.0$ (aromatic protons), 2.2-0.7 (br., polymer backbone); ${ }^{11} \mathrm{~B}$ NMR (128.286 MHz, $\left.\mathrm{CDCl}_{3}\right): \square=7\left(\mathrm{w}_{1 / 2}=750 \mathrm{~Hz}\right) ;{ }^{13} \mathrm{C}$ NMR data could not be obtained due to the poor solubility of the polymer. DSC measurement: $T_{\mathrm{g}}=221^{\circ} \mathrm{C}$ (onset).

Synthesis of Poly[4-(8-hydroxyquinolato-2-(5-hexylthienyl))borylstyrene] (PS-B5HTQ). A solution of PS-BBr prepared from $\mathrm{BBr}_{3}(3.27 \mathrm{~g}, 13.04 \mathrm{mmol})$ and PS-Si (2.00 g, ca. $11.34 \mathrm{mmol}$ repeat units; $\left.M_{w}=26,000 ; P D I=1.13\right)$ in $30 \mathrm{ml} \mathrm{CH}_{2} \mathrm{Cl}_{2}$ was treated with 5-hexyl-2trimethylstannylthiophene $(11.26 \mathrm{~g}, 34.02 \mathrm{mmol})$ and stirred for $24 \mathrm{~h}$. All volatile material was removed under high vacuum and the product was dried at $60{ }^{\circ} \mathrm{C}$ for $3 \mathrm{~h}$. Conversion to PSB5HT was confirmed by NMR spectroscopy; for PS-B5HT: ${ }^{1} \mathrm{H}$ NMR (499.893 $\mathrm{MHz}, \mathrm{CDCl}_{3}$ ): = 8.0-6.6 (aromatic protons), 3.0, 1.9, 1.6, 1.1 (hexyl-H), polymer backbone protons were not resolved; ${ }^{13} \mathrm{C}$ NMR (125.698 $\mathrm{MHz}, \mathrm{CDCl}_{3}$ ): $\square=158.1$ (Th-C5), 147 (br., Ph-C4), 143.0 (Th-C3), 142.0 (br., Ph-C1), 136.6 (Ph-C2,6), 126.6 (Th-C4 and Ph-C3,5), 47-42 (br., polymer backbone), 31.7, 31.6, 30.6, 29.1, 22.7, 14.2 (hexyl-C), Th-C2 was not resolved. The crude product was redissolved in $30 \mathrm{ml} \mathrm{CH}_{2} \mathrm{Cl}_{2}$ and used without further purification. A solution of 8hydroxyquinoline (3.96 g, $27.22 \mathrm{mmol}$ ) in $20 \mathrm{ml} \mathrm{CH}_{2} \mathrm{Cl}_{2}$ was then added dropwise. The mixture turned immediately yellow with green luminescence and was kept stirring for $24 \mathrm{~h}$. The reaction solution was concentrated under high vacuum to ca. $5 \mathrm{ml}$ and then precipitated into hexanes (ca. $500 \mathrm{ml}$ ). The product was reprecipitated twice from THF into methanol and subsequently from THF into hexanes. Polymer PS-B5HTQ was obtained as a yellow powder and dried at $60{ }^{\circ} \mathrm{C}$ under high vacuum for $24 \mathrm{~h}$ (yield: $4.0 \mathrm{~g}, 83 \%$ ). For PS-B5HTQ: ${ }^{1} \mathrm{H}$ NMR (499.893 MHz, $\mathrm{CDCl}_{3}$ ): $\square=8.6-6.0$ (br., all aromatic protons), 2.73, 1.59, 1.24, 0.83 (br., backbone and hexyl$\mathrm{H}$ ); ${ }^{13} \mathrm{C} \mathrm{NMR}\left(125.698 \mathrm{MHz}, \mathrm{CDCl}_{3}\right.$ ): $\square=158.3$ (Q-C8), 147.6 (Th-C5 and Ph-C4), 146-142 (very br., Th-C2 and Ph-C1), 139.6 (Q-C2), 139.2 (Q-C4), 136.9 (Q-C9), 132.0 (Q-C6), 131.7 
(Ph-C2,6), 130.2 (Th-C3), 128.2 (Q-C10), 127.2 (br., Ph-C3,5), 125.0 (Th-C4), 123.2 (Q-C3), 112.6 (Q-C5), 109.5 (Q-C7), 45-38 (br., $\left[\mathrm{CH}_{2} \mathrm{CH}\right]_{\mathrm{n}}$ ), 32.0, 31.8, 30.4, 29.2, 22.8, 14.3 (hexyl-C); ${ }^{11} \mathrm{~B}$ NMR $\left(128.286 \mathrm{MHz}, \mathrm{CDCl}_{3}\right): \square=5\left(\mathrm{w}_{1 / 2}=600 \mathrm{~Hz}\right)$. Static light scattering (Zimm plot; $d n / d c$ $=0.205 \mathrm{ml} / \mathrm{g}): M_{\mathrm{w}}=62,000 ; A_{2}=-6.707 \mathrm{e}-4 \mathrm{~mol} \bullet \mathrm{ml} / \mathrm{g}^{2}$. In-line GPC-LS: $M_{\mathrm{w}}=71,000, P D I=$ 1.04. Elemental analysis: calcd. C 76.23, H 6.63, N 3.29; found C 75.99, H 6.66, N 3.07. DSC measurement: $T_{\mathrm{g}}=146^{\circ} \mathrm{C}$ (onset).

Synthesis of Poly[4-(8-hydroxyquinolato-2-(3-hexylthienyl))borylstyrene] (PS-B3HTQ). A solution of PS-BBr prepared from $\mathrm{BBr}_{3}(0.85 \mathrm{~g} ; 3.40 \mathrm{mmol})$ and PS-Si $(0.50 \mathrm{~g}$, ca. $2.84 \mathrm{mmol}$ repeat units; $M_{w}=26,000 ; P D I=1.13$ ) in $30 \mathrm{ml} \mathrm{CH}_{2} \mathrm{Cl}_{2}$ was treated with 3-hexyl-2trimethylstannylthiophene $(3.66 \mathrm{~g}, 11.05 \mathrm{mmol})$ and stirred for $3 \mathrm{~d}$. All volatile material was removed under high vacuum and the product was dried at $60{ }^{\circ} \mathrm{C}$ for $3 \mathrm{~h}$. Conversion to PSB3HT was confirmed by NMR spectroscopy; for PS-B3HT: ${ }^{1} \mathrm{H}$ NMR (499.893 MHz, $\mathrm{CDCl}_{3}$ ): $\square$ = 7.8-6.2 (br., aromatic protons), 2.35, 1.40, 1.10, 0.94, 0.71 (br., hexyl-H), polymer backbone protons were not resolved; ${ }^{13} \mathrm{C}$ NMR (125.698 $\mathrm{MHz}, \mathrm{CDCl}_{3}$ ): $\square=153.7$ (br., Th-C3), 149 (br., Ph-C4), 141.8 (Th-C2), 141 (br., Ph-C1), 140.9 (Th-C5), 137.9 (br., Ph-C2,6), 133.9 (Th-C4), 130.8 (Th-C4), 127.0 (br., Ph-C3,5), $45-40$ (very br., polymer backbone), 32.4, 31.7, 29.4, 22.8, 14.3 (hexyl-C). The crude product was redissolved in $30 \mathrm{ml} \mathrm{CH}_{2} \mathrm{Cl}_{2}$ and used without further purification. A solution of 8-hydroxyquinoline $(0.83 \mathrm{~g}, 5.68 \mathrm{mmol})$ in $10 \mathrm{ml} \mathrm{CH} \mathrm{Cl}_{2}$ was then added dropwise. The mixture was kept stirring for $2 \mathrm{~d}$. The reaction solution was concentrated under high vacuum to ca. $5 \mathrm{ml}$ and then precipitated into hexanes (ca. $500 \mathrm{ml}$ ). The crude material was reprecipitated twice from THF into methanol and subsequently from THF into hexanes. Polymer PS-B3HTQ was obtained as a yellow powder and dried at $60{ }^{\circ} \mathrm{C}$ under high vacuum for $24 \mathrm{~h}$ (yield: $0.80 \mathrm{~g}, 67 \%$ ). ${ }^{1} \mathrm{H}$ NMR (499.893 $\mathrm{MHz}, \mathrm{CDCl}_{3}$ ): $\square=8.6-5.0$ (br., aromatic protons), 2.31, 1.07, 0.74 (br., backbone and hexyl-H); ${ }^{13} \mathrm{C} \mathrm{NMR}(125.698 \mathrm{MHz}$, $\mathrm{CDCl}_{3}$ ): $\square=158.5$ (Q-C8) 146.1 (Th-C3), 146-142 (very br. Ph-C4, Th-C2, Ph-C1), 140.0 (QC2), 139.0 (Q-C4), 137.2 (Q-C9), 132.6 (Q-C6), 131-130 (Th-C5 and Ph-C2,6), 128.2 (Q-C10), 127.5-126.5 (Ph-C3,5 and Th-C4), 123.1 (Q-C3), 112.5 (Q-C5), 109.5 (Q-C7), 45-39 (very br., polymer backbone), 31.9, 31.0, 30.6, 29.5, 22.8, 14.4 (hexyl-C); ${ }^{11} \mathrm{~B}$ NMR (128.286 MHz, $\left.\mathrm{CDCl}_{3}\right): \square=5\left(\mathrm{w}_{1 / 2}=500 \mathrm{~Hz}\right)$. Static light scattering (Zimm plot; $\left.d n / d c=0.201 \mathrm{ml} / \mathrm{g}\right): M_{\mathrm{w}}=$ 64,000; $A_{2}=-9.499 \mathrm{e}-4 \mathrm{~mol} \bullet \mathrm{ml} / \mathrm{g}^{2}$. In-line GPC-LS: $M_{\mathrm{w}}=66,000, P D I=1.06$. Elemental analysis: calcd. C 76.23, H 6.63, N 3.29; found C 76.17, H 6.65, N 3.25. DSC measurement: $T_{\mathrm{g}}=$ $146{ }^{\circ} \mathrm{C}$ (onset).

References:

(1) Qin, Y.; Cheng, G.; Sundararaman, A.; Jäkle, F. J. Am. Chem. Soc. 2002, 124, 12672

(2) Michalitsch, R.; Elkassmi, A.; Yassar, A.; Garnier, F. J. Heterocyclic Chem. 2001, 38, 649.

(3) (a) Barbarella, G.; Bongini, A.; Massimo, Z. Macromolecules 1994, 27, 3039;

(b) Trznadel, M.; Pron, A.; Zagorska, M.; Chrzaszcz, R.; Pielichowski, J.

Macromolecules 1998, 31, 5051.

(4) Aue, W. P., Bartholdi, E., and Ernst, R. R. J. Chem. Phys. 1976, 64, 2229.

(5) Bax, A., and Freeman, R. J. Magn. Reson. 1981, 44, 542. 
(6) Muller, L. J. Am. Chem. Soc. 1979, 101, 4481.

(7) Bax, A., and Subramanian, S. J. Magn. Reson. 1986, 67, 565.

(8) Redfield, A. G., and Kunz, S. D. J. Magn. Reson. 1975, 19, 250.

(9) Marion, D., and Wuthrich, K. Biochem. Biophys. Res. Commun. 1983, 113, 967.

(10) States, D. J., Haberkorn, R. A., and Ruben, D. J. J. Magn. Reson. 1982, 48, 286.

(11) von Kienlin, M., Moonen, C. T. W., van der Toorn, A., and van Zijl, P. C. M. J. Magn. Reson. 1991, 93, 432.

(12) Garbow, J. R., Weitekamp, D. P., and Pines, A. Chem. Phys. Lett. 1982, 93, 504.

(13) Shaka, A. J., Barker, P. B., and Freeman, R. J. Magn. Reson. 1985, 64, 547.

(14) Hoult, D. I. J. Magn. Reson. 1983, 51, 110

(15) Otting, G., Widmer, H., Wagner, G., and Wuthrich, K. J. Magn. Reson. 1986, 66, 187.

Figure S1. Absorption and emission spectra of PS-B3HTQ and M-B3HTQ in THF

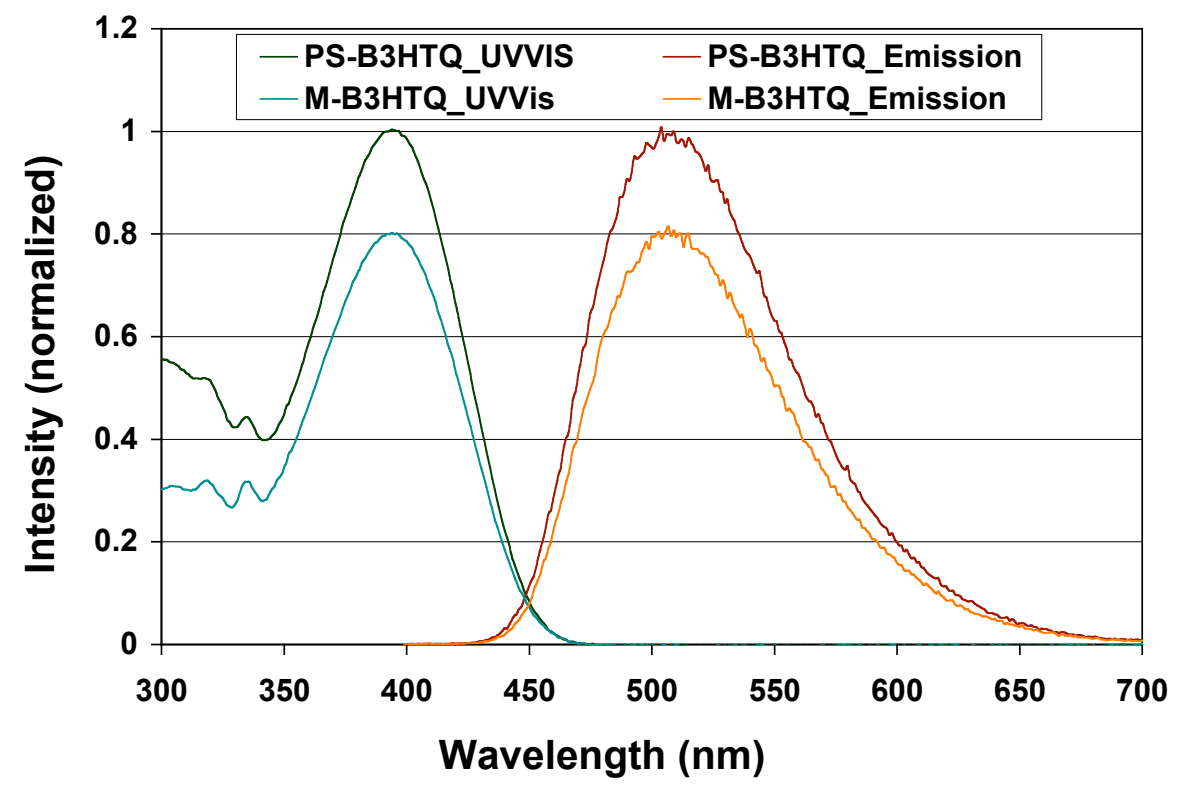


Figure S2. Aromatic region of the gCOSY NMR spectrum of M-B5HTQ in $\mathrm{CDCl}_{3}$.

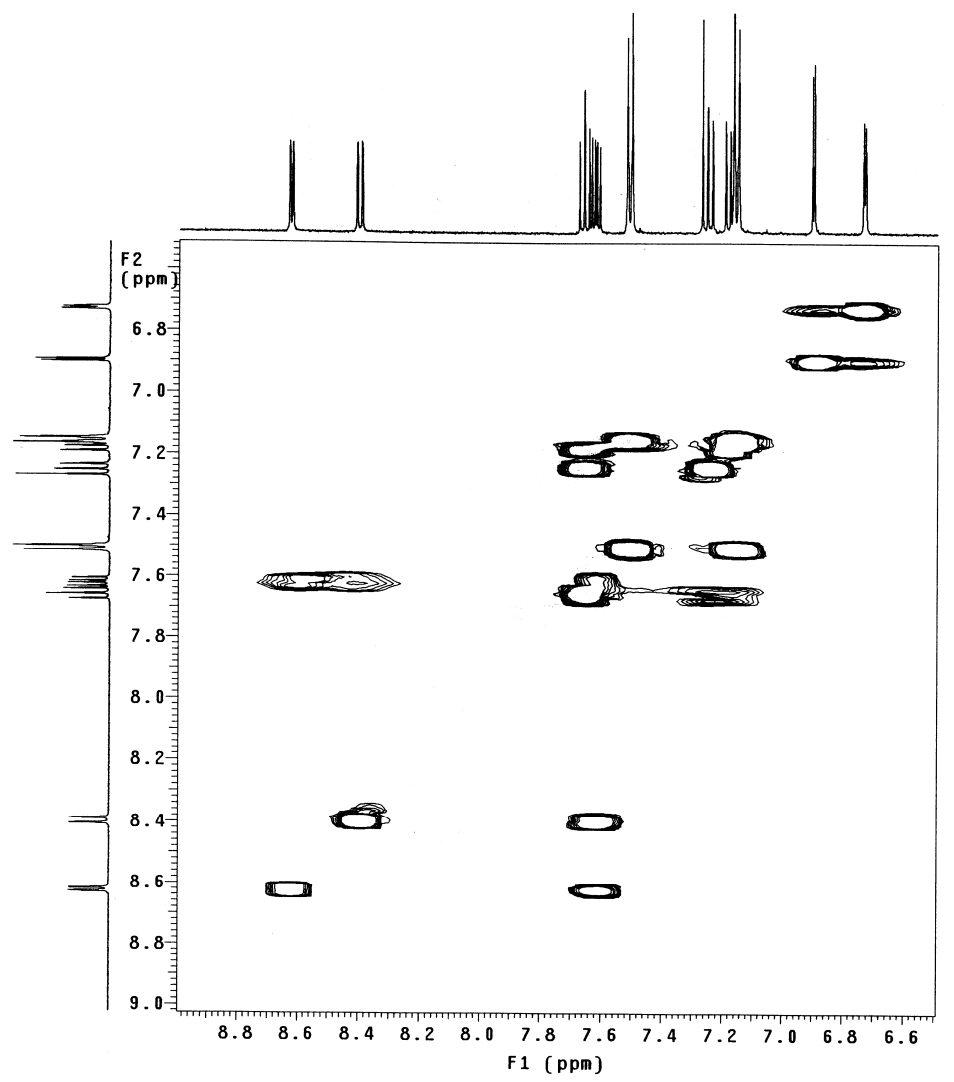

Figure S3. Region of aromatic C-H resonances of the HMQC NMR spectrum of M-B5HTQ in $\mathrm{CDCl}_{3}$.

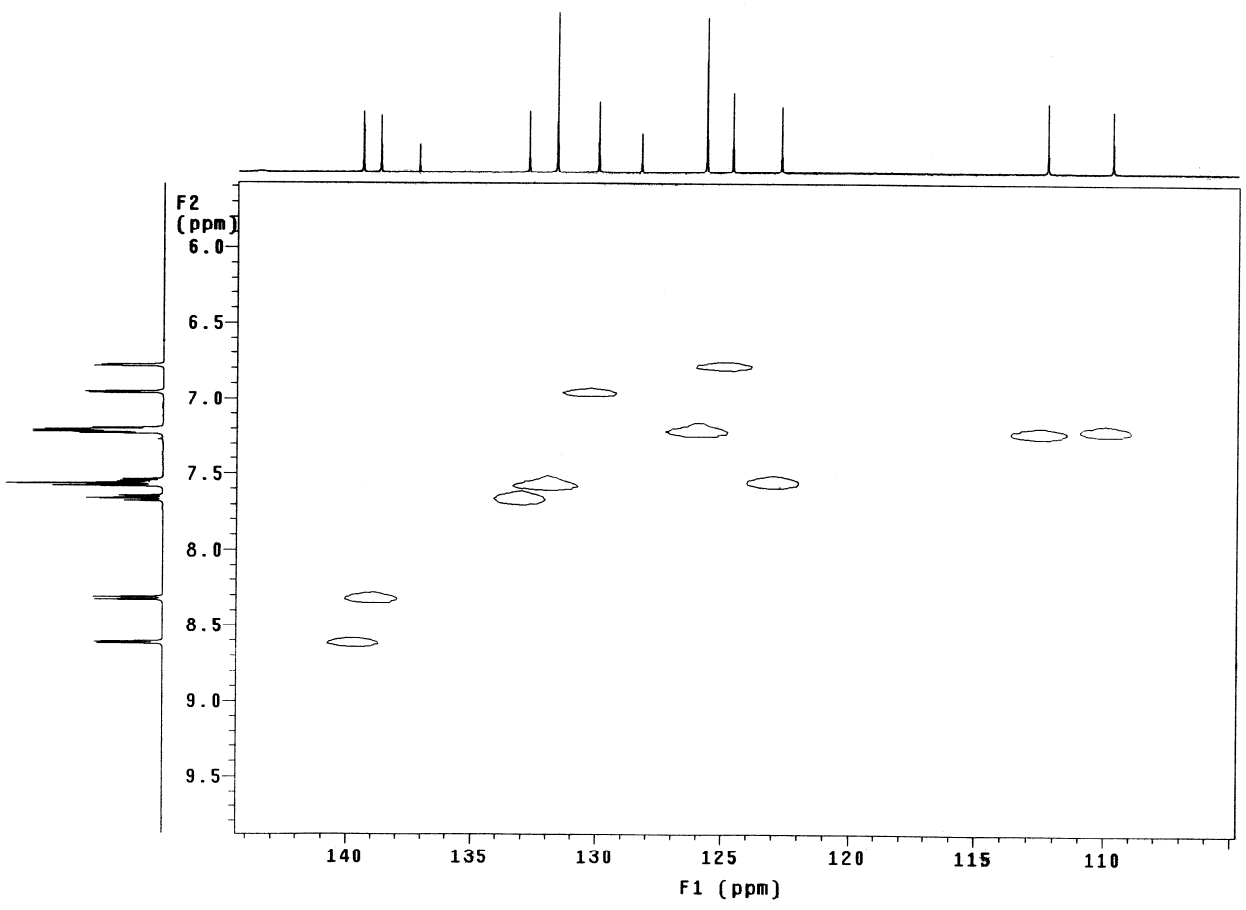


Figure S4. Luminescence Lifetime Measurements for PS-B5HTQ and M-B5HTQ in THF.
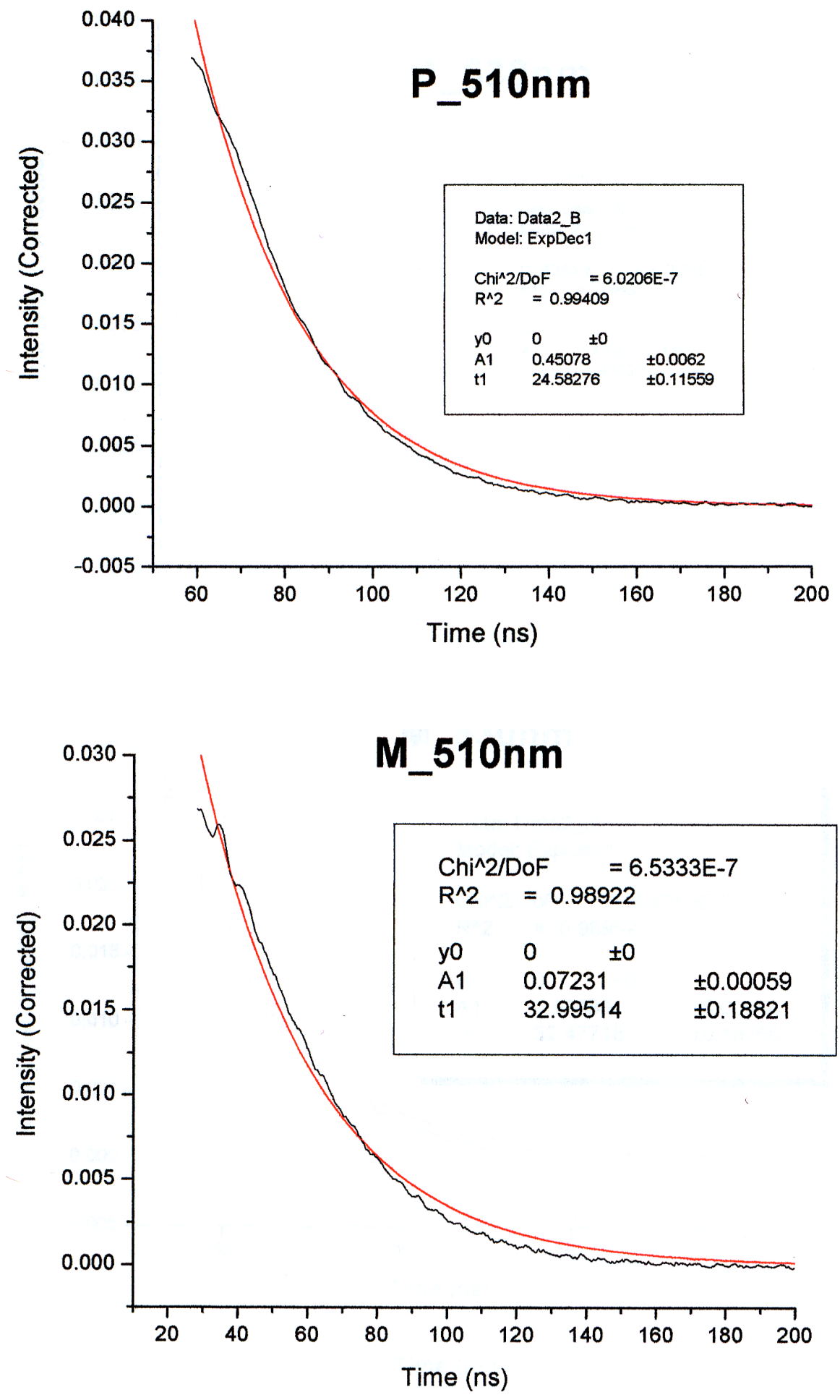